\title{
Musculoskeletal involvement of COVID-19: review of imaging
}

\author{
Santhoshini Leela Ramani ${ }^{1}$ • Jonathan Samet ${ }^{2}$ - Colin K. Franz ${ }^{3,4}$. Christine Hsieh ${ }^{5}$ Cuong V. Nguyen ${ }^{6}$. \\ Craig Horbinski ${ }^{7}$. Swati Deshmukh ${ }^{2}$
}

Received: 10 January 2021 / Revised: 1 February 2021 / Accepted: 2 February 2021 / Published online: 18 February 2021

(C) ISS 2021

\begin{abstract}
The global pandemic of coronavirus disease 2019 (COVID-19) has revealed a surprising number of extra-pulmonary manifestations of severe acute respiratory syndrome coronavirus 2 (SARS-CoV-2) infection. While myalgia is a common clinical feature of COVID-19, other musculoskeletal manifestations of COVID-19 were infrequently described early during the pandemic. There have been emerging reports, however, of an array of neuromuscular and rheumatologic complications related to COVID-19 infection and disease course including myositis, neuropathy, arthropathy, and soft tissue abnormalities. Multimodality imaging supports diagnosis and evaluation of musculoskeletal disorders in COVID-19 patients. This article aims to provide a first comprehensive summary of musculoskeletal manifestations of COVID-19 with review of imaging.
\end{abstract}

Keywords COVID-19 $\cdot$ SARS-CoV-2 $\cdot$ Musculoskeletal $\cdot$ Neuromuscular $\cdot$ MR $\cdot$ Ultrasound

\section{Introduction}

Severe acute respiratory syndrome coronavirus 2 (SARSCoV-2) was first identified in Wuhan, China, in December 2019 and rapidly spread throughout the world. Coronavirus disease 2019 (COVID-19) was officially declared a pandemic on March 11, 2020, by the World Health Organization, with over 90 million cases worldwide as of January 2021 [1-3]. An

Swati Deshmukh

swati.deshmukh@northwestern.edu

1 Northwestern University Feinberg School of Medicine, 420 E Superior St, Chicago, IL 60611, USA

2 Department of Radiology, Northwestern University Feinberg School of Medicine, 420 E Superior St, Chicago, IL 60611, USA

3 Shirley Ryan Ability Lab (Formerly the Rehabilitation Institute of Chicago), 355 E Erie St, Chicago, IL 60611, USA

4 Departments of Physical Medicine and Rehabilitation and Neurology, Northwestern University Feinberg School of Medicine, 420 E Superior St, Chicago, IL 60611, USA

5 Department of Rheumatology, Northwestern University Feinberg School of Medicine, 420 E Superior St, Chicago, IL 60611, USA

6 Department of Dermatology, Northwestern University Feinberg School of Medicine, 420 E Superior St, Chicago, IL 60611, USA

7 Division of Pathology, Department of Medicine, Northwestern University Feinberg School of Medicine, 420 E Superior St, Chicago, IL 60611, USA estimated 5\% of COVID-19 patients have severe symptoms that require intensive care [4]. Older age and comorbidities such as cardiovascular disease, diabetes mellitus, and obesity are risk factors for developing severe disease [2]. Potential therapeutic measures for acute symptomatic COVID-19 patients include supplemental oxygen and mechanical ventilation, corticosteroids, and thromboembolic prophylaxis [4]. Currently, treatment recommendations and preventative measures are continually evolving.

SARS-CoV-2 is an RNA virus with a viral structural spike (S) protein that binds to the angiotensin-converting enzyme 2 (ACE2) receptor on human cells (Fig. 1). There is high expression of the ACE2 receptor in lung epithelial cells as well as in the heart, kidney, pancreas, spleen, gastrointestinal system, bladder, cornea, and blood vessels [2, 4]. The ACE2 receptor is also found in the central and peripheral nervous systems and in skeletal muscle [5, 6]. Viral replication within human host cells is followed by viral release through cell destruction $[2,4]$. In addition, SARS-CoV-2 activates an inflammatory response (both innate and adaptive immune responses) which can result in a cytokine storm and ultimately multi-organ injury $[2,4]$.

Primarily a respiratory disease, COVID-19 may manifest in multiple ways, ranging from asymptomatic presentation to mild upper respiratory tract infection symptoms to acute respiratory distress syndrome (ARDS). Numerous extrapulmonary manifestations are now known to occur with SARS-CoV-2 infection including gastrointestinal symptoms, 
Fig. 1 Illustration of SARS-CoV2 infection

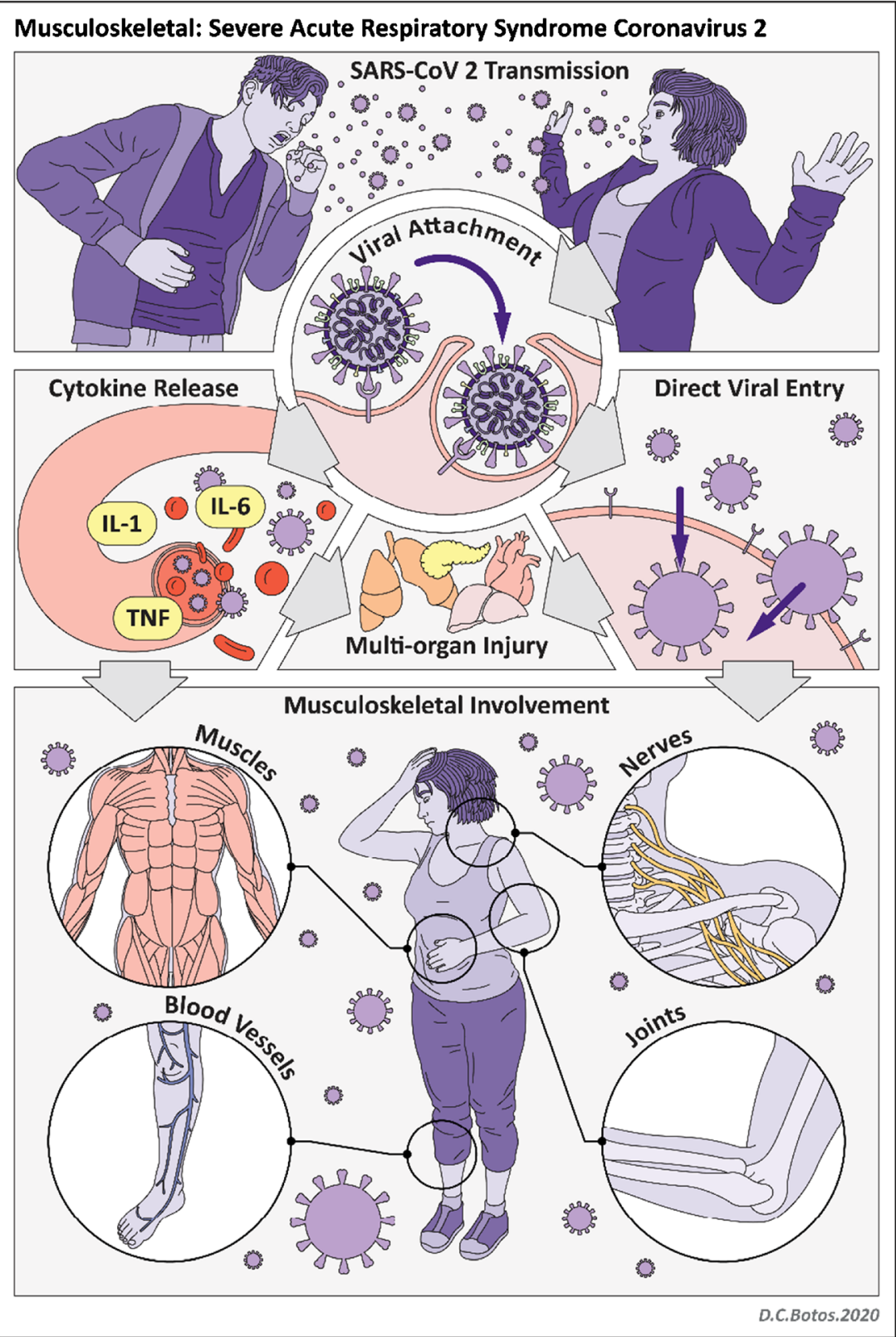

kidney and liver injury, myocardial dysfunction and acute coronary syndromes, neurologic complications, and dermatologic findings. While myalgia is a common clinical feature of COVID-19, other musculoskeletal manifestations of COVID19 were infrequently described early during the pandemic [2-4]. As the global numbers of COVID-19 patients and survivors rose, however, there have been increasing reports of neuromuscular and rheumatologic complications related to both the virus and treatment/hospital course [5]. Imaging, including magnetic resonance (MR) imaging, computed tomography (CT), and ultrasound, can support diagnosis and evaluation of musculoskeletal manifestations and iatrogenic complications of COVID-19 (Table 1). In this article, we review mechanisms and imaging features of COVID-19-related disorders of the musculoskeletal system - specifically, of muscles, nerves, joints, soft tissues, and bone.

\section{Muscle}

Myalgia, defined as muscle aches and pain, has been frequently reported in COVID-19 patients with a prevalence ranging from 11 to $50 \%$ in large cohort studies [5]. Several case reports have described myositis and rhabdomyolysis in COVID19 patients, both as a late complication and as a presenting symptom [3, 5, 7]. Rare cases of SARS-CoV-2 triggering necrotizing autoimmune myositis have been described [6]. Mechanisms of muscular involvement in COVID-19 are not 
Table 1 Imaging of musculoskeletal involvement in COVID-19

\begin{tabular}{lll}
\hline Organ system & Imaging modalities & Imaging findings \\
\hline Muscle & MRI +/- contrast & Muscle edema, necrosis \\
& Ultrasound & Duscle atrophy \\
Nerve & MR neurography & Nerve enlargement, signal hyperintensity, loss of fascicular architecture \\
& $+/-$ muscle denervation \\
& High-resolution ultrasound & Nerve enlargement, hypoechogenicity, loss of fascicular architecture \\
Joints & MRI +/- contrast & Joint effusion with enhancement, +/- erosions \\
Soft tissues & Ultrasound with Doppler & Synovitis, hyperemia a \\
Bone & MRI, CT, ultrasound & Hematomas, gangrene, "COVID toes," atypical pressure ulcers from prone positioning \\
& Radiography, CT, MRI & Osteoporosis, osteonecrosis \\
\hline
\end{tabular}
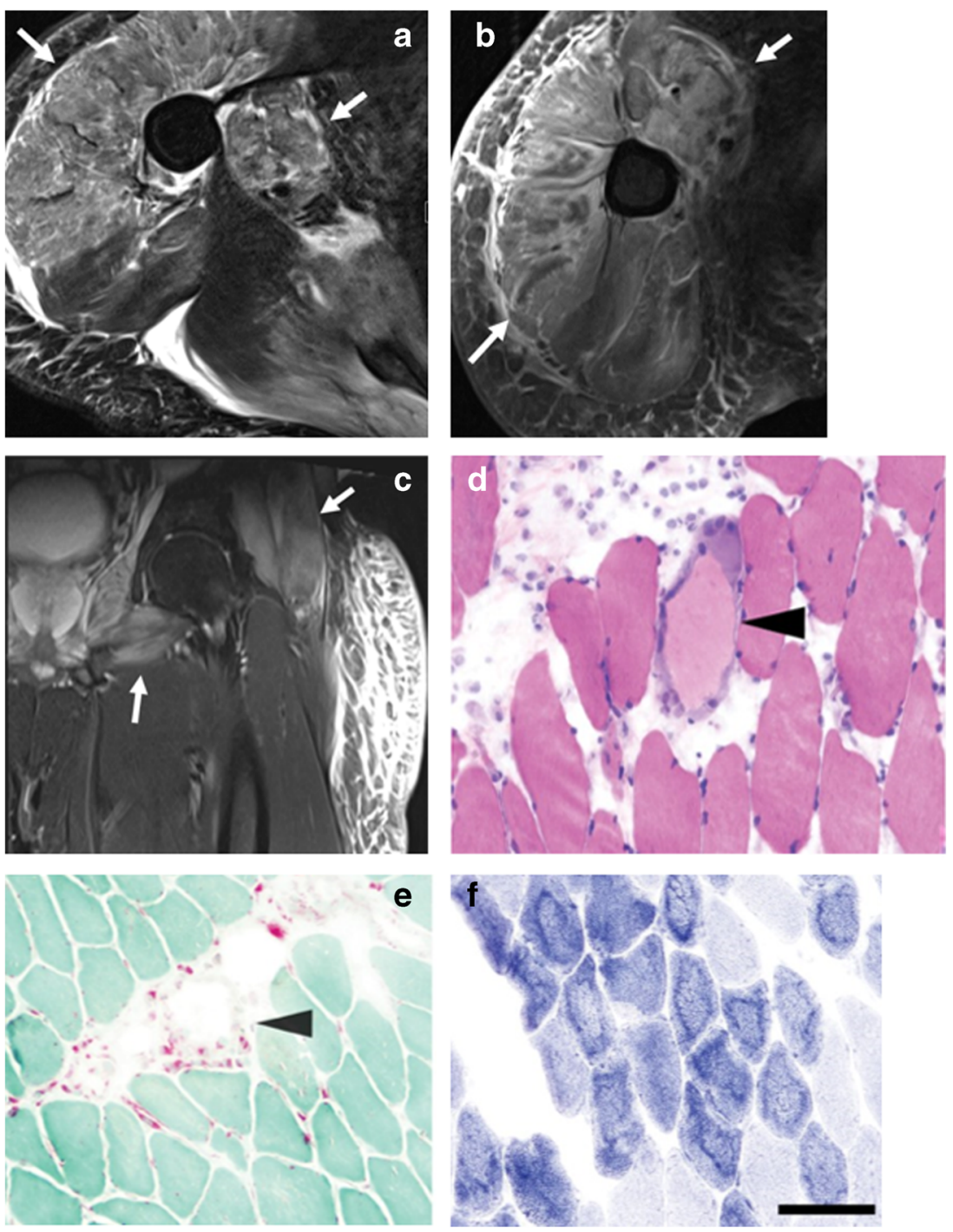

Fig. 2 A 44-year-old male with incidental positive COVID-19 test obtained prior to eye surgery. He did not have any respiratory symptoms and was surprised by the positive test. In the following 2-3 weeks, he developed progressive weakness and swelling requiring hospitalization. MR imaging of the upper and lower extremities with intravenous contrast was performed. a Axial T2-weighted fat-saturated and $\mathbf{b}$ axial post-contrast T1-weighted fat-saturated images demonstrate diffuse edema and enhancement of the proximal right upper extremity musculature (arrows). c Coronal T2-weighted fat-saturated image of the left lower extremity demonstrates muscle edema of the proximal muscles/limb girdle

(arrow) with sparing of the distal muscles. Subcutaneous soft tissue edema is noted. Biopsy of the deltoid muscle was performed. d Histopathologic findings included scattered pale degenerating myofibers, surrounded by macrophages (arrow) on H\&E stain. e Acid phosphatase highlighted the macrophages (arrow). f NADH stain showed an unusual ring-like pattern to the myofibrillar architecture (scale bar $=100 \mu \mathrm{m}$ ). The patient was diagnosed with post-infectious inflammatory necrotizing myositis and discharged on oral prednisone with subsequent clinical improvement and decrease in CK levels on follow-up 
fully understood. Hematogenous spread and direct invasion of skeletal muscle by SARS-CoV-2 through the ACE2 receptor have been proposed [5, 6]. Immune-mediated mechanisms are an alternate and more widely accepted theory of muscle involvement of SARS-CoV-2, thought to be secondary to an inflammatory response with cytokine storming and activation of immune cells. Suggested mechanisms of immune-mediated muscle damage include immune complex deposition, myotoxic cytokine release, and injury secondary to homology between human muscle cells and viral antigens $[5,6]$.

Myositis broadly refers to inflammation of muscles and is associated with SARS-CoV-2 as well as other viral infections such as influenza A/B, hepatitis, and HIV [8]. Rhabdomyolysis is a complication of myositis involving infarction of muscle (myonecrosis) and high levels of myoglobin in the blood (myoglobinemia). Rhabdomyolysis is a life-threatening condition that can lead to acute kidney failure, compartment syndrome, and intravascular coagulation [3,9]. Clinical findings of myositis/rhabdomyolysis generally include myalgia/weakness and elevated creatine phosphokinase kinase levels, both of which have been reported in COVID-19 patients with myositis/rhabdomyolysis [5, 8]. Electrodiagnostic studies, such as electromyography (EMG), and nerve conduction studies can be helpful to confirm a myopathic process and exclude mimickers such as motor neuron disease [10]. Imaging can support diagnosis and delineate sites for muscle biopsy, which is the gold standard for diagnosis [3, 9]. MR imaging is the modality of choice, preferably
Fig. 3 A 53-year-old male with prolonged hospitalization for COVID-19 ARDS who developed multiple peripheral nerve injuries during his hospital course and presented with clinical concern of right phrenic nerve palsy. a Ultrasound of the right hemidiaphragm (arrowheads) at expiration and $\mathbf{b}$ inspiration demonstrates little change in thickness of the hemidiaphragm muscle, compatible with decreased contractility function. There is no evidence of right hemidiaphragm muscle atrophy. $\mathbf{c}$ High-resolution ultrasound of the phrenic nerve (arrow, calipers) demonstrates normal size and echogenicity. Differential diagnosis for hemidiaphragm paralysis includes critical illness myopathy, ventilator-induced diaphragm dysfunction, and the hypothetical plausibility of virus-related myopathy
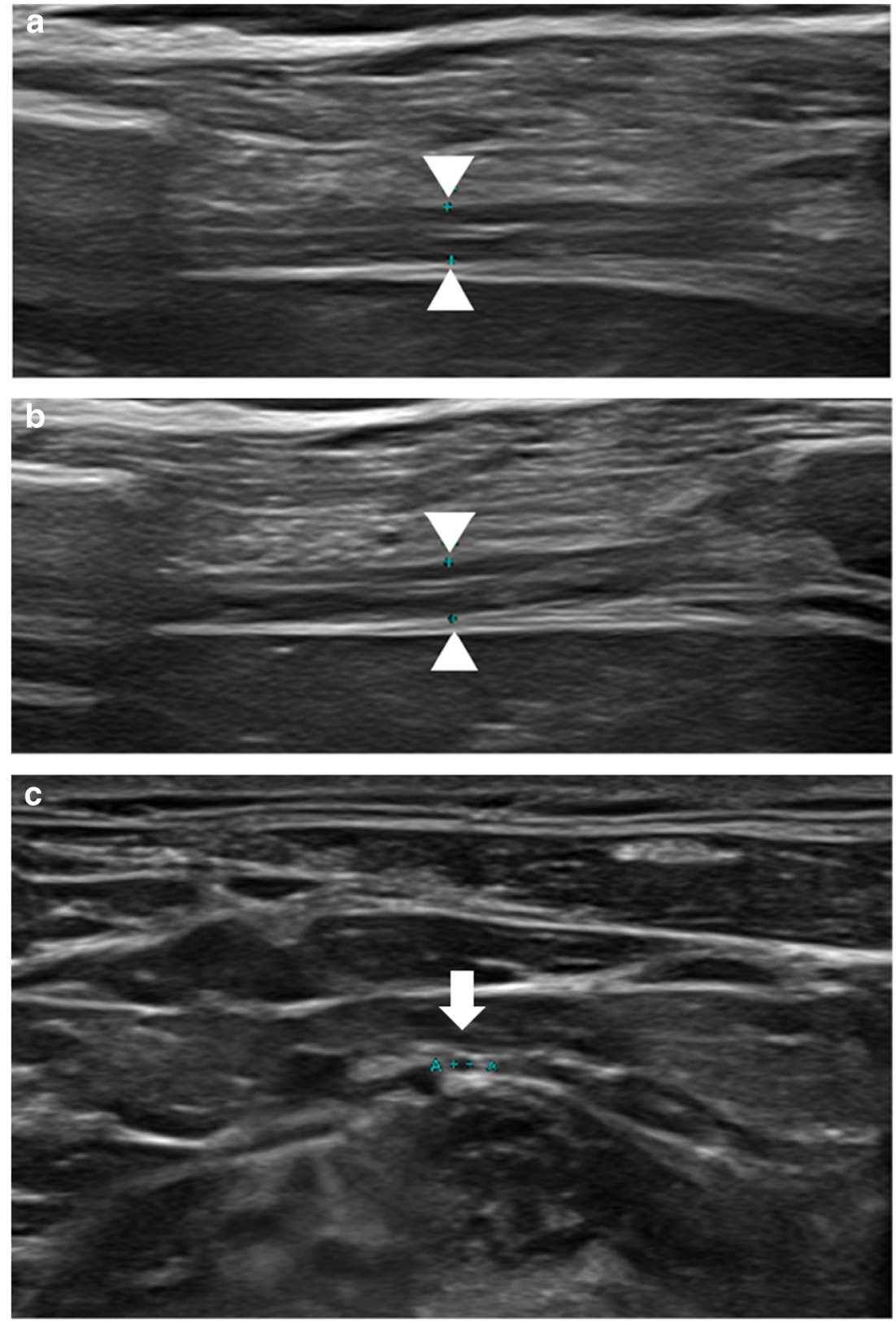
with either a $1.5-\mathrm{T}$ or 3.0-T magnet and inclusive of multiplanar fluid-sensitive and anatomic sequences. Findings of myositis include muscle edema, identified as increased signal intensity on T2-weighted or short tau inversion recovery (STIR) sequences (Fig. 2) [9]. Patterns of disease include homogeneous hyperintense signal and enhancement (type 1) and heterogeneous hyperintense signal and rim enhancement (type 2) [3]. In severe disease, areas of necrosis or loss of normal muscle architecture may be seen. A distinguishing finding of myonecrosis is the "stipple sign" with foci of enhancement in a rim-enhancing area of non-enhancing muscle tissue [3]. Intramuscular hemorrhage may be present, identified as T1 hyperintense signal or blooming artifact on gradient echo sequences [8].

Differential diagnosis for muscle edema on MRI in hospitalized COVID-19 patients includes critical illness myopathy, which is a common acquired condition in intensive care unit (ICU) patients that has been reported in COVID-19 patients requiring ICU care and has also been associated with corticosteroid use $[6,11]$. Clinical presentation of critical illness myopathy includes symmetric and generalized weakness or acute flaccid quadriplegia [6]. Critical illness myopathy is a primary myopathy with non-specific imaging findings of multifocal muscle edema and atrophy [11]. Findings of necrosis are not present, in contrast to COVID-19-related rhabdomyolysis/ myonecrosis. Clinical and imaging features of critical illness myopathy in COVID-19 are not distinctive from nonCOVID-19 patients, although the degree of spontaneous activity on electrodiagnostic studies was found to be strikingly severe in one study of critically ill COVID-19 patients [12].

Diaphragm muscle dysfunction can occur in COVID-19 patients secondary to critical illness myopathy, ventilatorinduced diaphragm dysfunction, or phrenic nerve injury, possibly from placement of chest support devices. Hypothetically, direct neuromuscular involvement of the SARS-CoV-2 virus may contribute to diaphragm dysfunction $[13,14]$. A recent autopsy study, in fact, found ACE2 expression in the human diaphragm and SARS-CoV-2 viral RNA in a subset of COVID-19 patients, with increased fibrosis of the diaphragm muscle and a unique myopathic phenotype compared to control ICU patients [15]. Diaphragm dysfunction can lead to deteriorating respiratory status and/or difficulty in weaning from mechanical ventilation [13, 14]. Imaging is helpful for diagnosis and monitoring of diaphragm dysfunction in COVID-19 patients. The fluoroscopy sniff test offers a quick and real-time assessment of diaphragm excursion. Ultrasound contributes additional information including the presence or absence of diaphragm muscle atrophy, calculation of the muscle thickening ratio with respiration, and evaluation of excursion with $\mathrm{M}$ mode imaging. High-resolution ultrasound can also evaluate the phrenic nerve in the region of the neck, which may aid in differentiation of neuropathic versus myopathic causes of diaphragm dysfunction (Fig. 3).
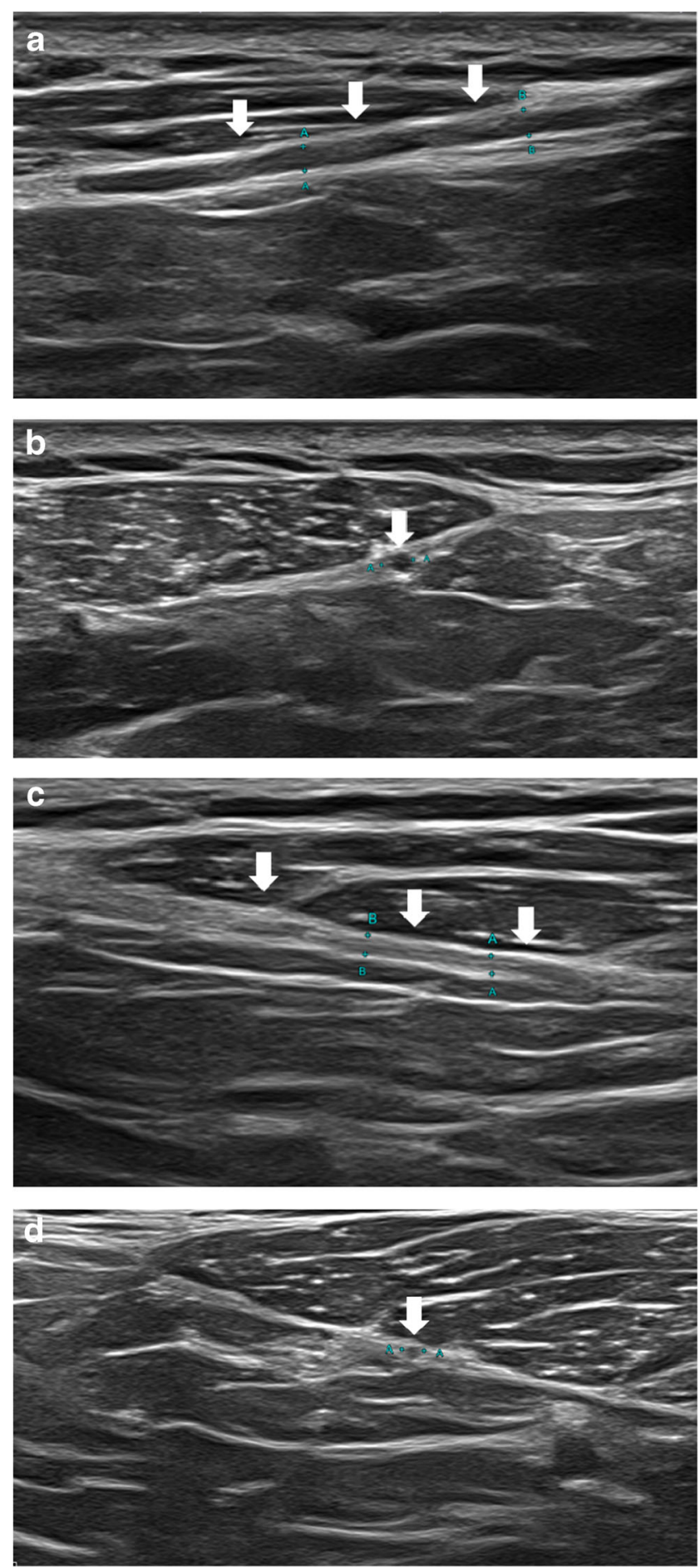

Fig. 4 A 60-year-old male with prolonged hospitalization for COVID-19 ARDS including prone positioning who developed multiple peripheral nerve injuries during his hospital course and presented with severe neck and shoulder pain. Clinical concern was for right spinal accessory nerve injury. a Longitudinal and b transverse high-resolution ultrasound demonstrates thickening and hypoechogenicity of the right spinal accessory nerve (arrows). c Longitudinal and $\mathbf{d}$ transverse high-resolution ultrasound of the normal left spinal accessory nerve (arrows) is shown for comparison 
Long-term muscular sequelae of COVID-19 include sarcopenia and cachexia which have been described in COVID-19 patients with prolonged illness [5]. Sarcopenia (or myopenia) is defined as muscle loss, typically associated with aging although other contributing factors include inactivity and poor nutrition [9]. Cachexia entails muscle wasting secondary to chronic illness. MR imaging findings of muscle atrophy, as seen in sarcopenia and cachexia, include decreased muscle size and fat infiltration [9].

\section{Nerves}

Peripheral neuropathy in the setting of COVID-19 has increasingly been reported $[5,6,12,16-20]$. Mechanisms of nerve involvement in COVID-19 are not fully understood. In one autopsy series of COVID-19 patients, SARS-CoV-2 viral proteins were detected in the medulla oblongata and cranial nerves IX and X [21]. The hypothetical ability of SARS$\mathrm{CoV}-2$ to act as a new neuropathogen and directly invade peripheral nerves via the ACE2 receptor warrants further investigation $[5,6,21,22]$. The concept of "molecular mimicry" is an alternative theory that may account for peripheral nerve injury, given similarities between SARS-CoV-2 surface glycoproteins and glycoconjugates in human nervous tissues [5,
6]. Direct cytotoxic effects of the virus on peripheral nerves have also been postulated [5].

Multiple case reports of Guillain-Barre syndrome (GBS) secondary to COVID-19 have been published, with symptoms emerging 3-4 weeks after onset of COVID-19 symptoms [5, $6,16,17]$. GBS is typically a demyelinating polyneuropathy that manifests as acute ascending paralysis. Facial weakness and cranial neuropathies may also occur. Miller-Fisher syndrome is a GBS variant characterized by gait ataxia, areflexia, and ophthalmoplegia, and has also been reported in association with COVID-19 [5, 6, 16]. MR imaging findings of GBS and GBS variants include signal hyperintensity, enlargement, and mild to moderate contrast enhancement of the nerve roots/ plexus and cauda equina. In chronic inflammatory demyelinating polyneuropathy (a chronic analog to GBS), contrast enhancement is usually not present [23].

Aside from GBS and its variants, post-infectious immunemediated neuropathies secondary to COVID-19 have been rarely reported to date $[5,17]$. A single case report of an otherwise healthy 17-year-old patient describes ParsonageTurner syndrome (neuralgic amyotrophy) in the setting of positive COVID-19 serum IgG antibodies [18]. Imaging features of post-infectious peripheral neuropathy are not specific to the infectious agent and include nerve enlargement, loss of fascicular architecture, and signal hyperintensity and
Fig. 5 A 37-year-old female with history of hospitalization for COVID-19 complicated by sacral ulcers presenting with left sciatic mononeuropathy. a Coronal postcontrast (for vascular suppression) T2 SPACE and b axial T2weighted fat-saturated images of the left femur demonstrate diffuse signal hyperintensity of the sciatic nerve (arrow). Subtle asymmetric fatty atrophy and edema of the posterior muscle compartment of the thigh (arrowhead) is suggestive of early denervation. c Axial T1-weighted anatomic image identifies the sciatic nerve (arrow). Based on MR imaging, she was referred to a neuromuscular specialist whose clinical evaluation supported the diagnosis of compressive sciatic neuropathy acquired during her critical illness
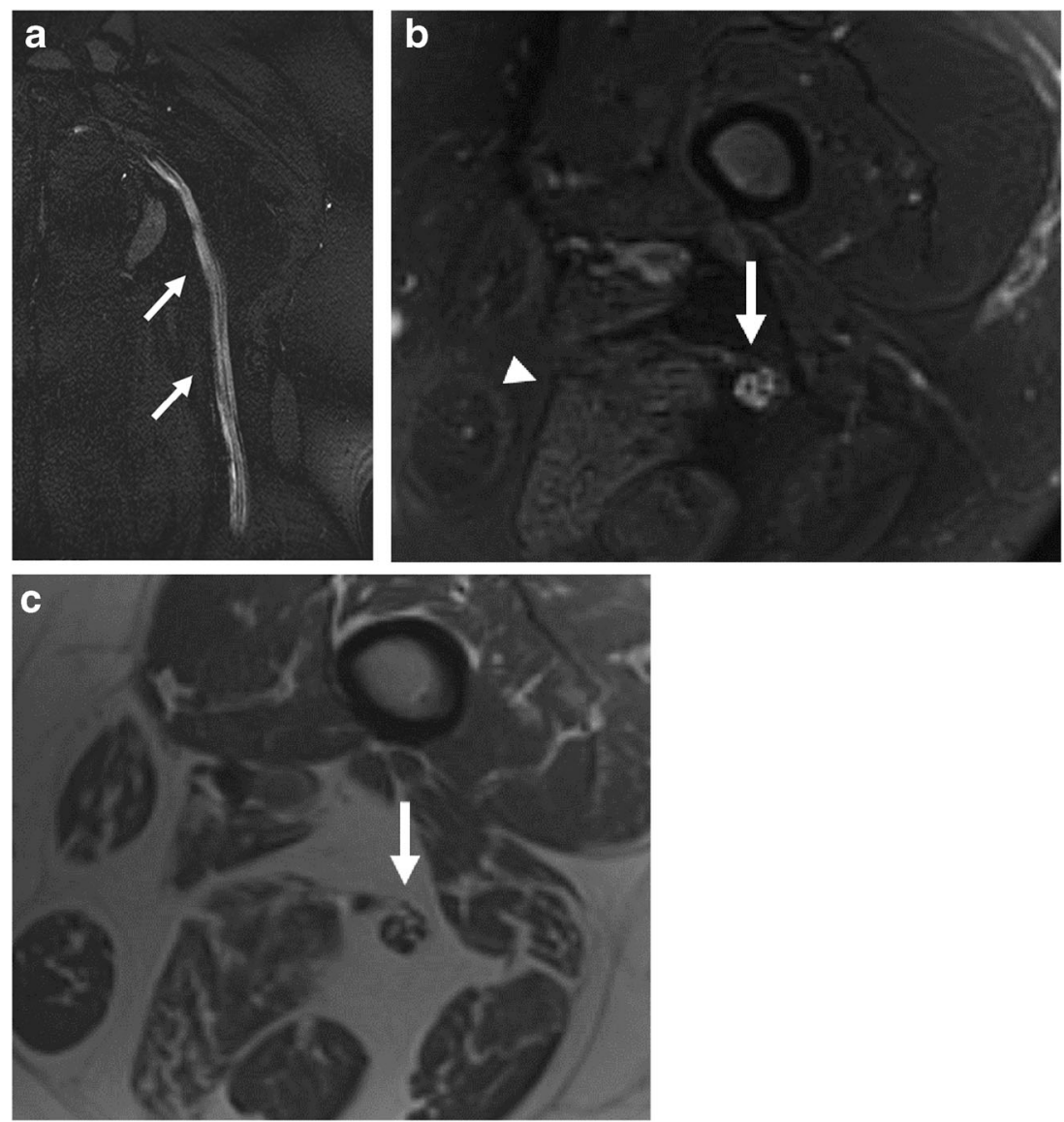
hypoechogenicity on MR neurography and ultrasound respectively. Secondary findings of muscle denervation may be present and entail muscle edema in the subacute phase and fatty atrophy in the chronic setting [24-26].

Iatrogenic peripheral neuropathy has been reported in the setting of COVID-19 with a higher-than-expected incidence, raising the possibility that the SARS-CoV-2 virus may predispose peripheral nerves to injury via theoretical mechanisms that have yet to be firmly established [12, 19, 20]. COVID-19 patients may plausibly be more susceptible to iatrogenic nerve injuries secondary to a virus-induced state of hyperinflammation or overlapping comorbidities that predispose to both nerve injury and severe COVID-19 symptoms that necessitate hospitalization [20]. Prone positioning for optimization of oxygenation in hospitalized COVID-19 patients has been widely adopted; while life-saving, these measures may have adverse effects on the peripheral nervous system [27]. Prone positioning and repositioning maneuvers during hospital course can lead to stretch/traction injuries of peripheral nerves. Compression injuries of peripheral nerves can also occur secondary to external compression with prolonged prone positioning or internal compression from an intramuscular hematoma. Critical illness polyneuropathy is a symmetric sensory-motor axonal polyneuropathy that occurs in patients with prolonged hospitalization and has been reported in association with COVID-19 ICU stay [12]. To date, there are no distinguishing imaging features of COVID-19-related peripheral neuropathy. General imaging findings of neuropathy that may be seen in iatrogenic peripheral nerve injury include nerve signal hyperintensity and hypoechogenicity on MR neurography and ultrasound, respectively, nerve enlargement, and loss of fascicular architecture (Figs. 4 and 5). Critical illness polyneuropathy is classically bilateral and symmetric whereas the first clue of a positioningrelated neuropathy in post-ICU patients is asymmetric involvement of a peripheral nerve dermatome and/or myotome. Segmental nerve narrowing secondary to mass effect can be seen in hematoma-related compression neuropathy. In the subacute and chronic settings after peripheral nerve injury, evidence of muscle denervation may be seen in a distribution corresponding to the affected nerve [24-26]. Imaging can serve as an alternate or adjuvant test to electrodiagnostic studies for COVID-19 patients with peripheral nerve injury.

\section{Joints}

Coronaviruses, in general, are more associated with arthralgia and myalgia rather than clinical arthritis [28]. Arthralgia has been reported as a symptom of COVID-19, occurring in $2.5 \%$ of patients according to a single study that separated arthralgia
Fig. 6 A 72-year-old female with history of rheumatoid arthritis that had been dormant for over 2 years who experienced multi-joint rheumatoid arthritis flair after contracting COVID-19 (with documented positive testing). Of note, she experienced only mild symptoms with her acute SARSCoV-2 infection. a Coronal, b sagittal, and $\mathbf{c}$ axial post-contrast T1-weighted images of the right shoulder demonstrate moderate enhancing synovitis with extensive low signal intensity debris (arrows). Her symptoms improved following intra-articular steroid injection
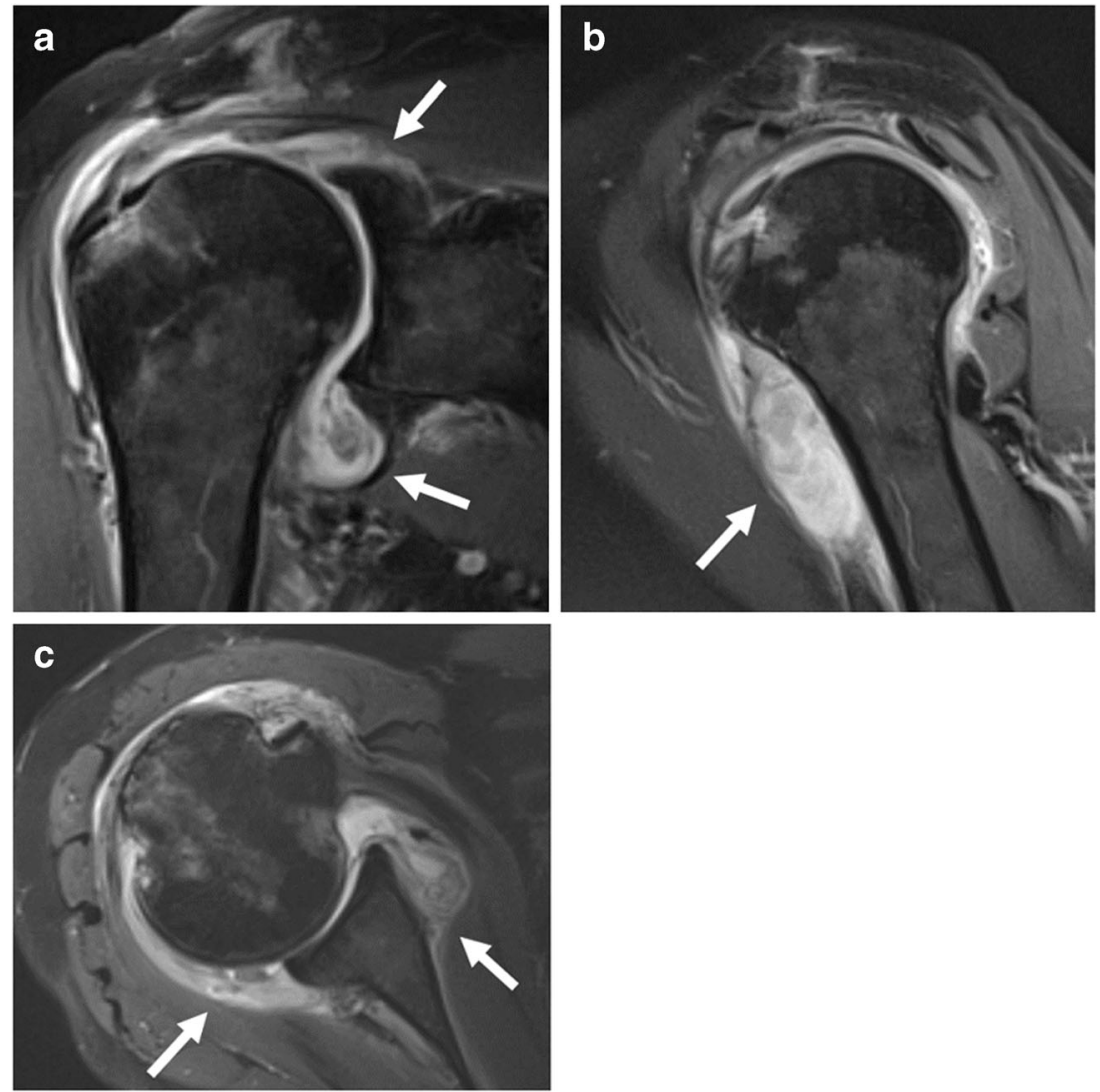
from myalgia as an independent symptom [29]. To date, there have been only several cases of acute clinical arthritis secondary to COVID-19 reported in the literature, some of which demonstrate features suggestive of reactive arthritis or crystalline arthritis rather than viral arthritis, emphasizing the importance of evaluating for other causes of arthropathy in these scenarios [29-34]. Virus-induced arthritis can be challenging to confirm, but findings that suggest viral arthritis include onset of arthralgia within a few weeks following viral infection, a self-limiting course, and a good response to NSAIDs. There are no current imaging findings distinct to COVID-19. Serological testing and fluid sampling are helpful to exclude other potential etiologies of arthropathy including septic arthritis, rheumatoid arthritis, psoriatic arthritis, crystalline arthritis, Lyme disease, systemic lupus erythematous, and reactive arthritis secondary to other infections such as chlamydia/ gonorrhea [30, 31].

Various chronic rheumatologic diseases triggered by SARS-CoV-2 have been reported, including systemic lupus erythematosus, dermatomyositis, Graves' disease, rheumatoid arthritis, and psoriatic spondyloarthritis [35-40]. Inflammatory arthropathies may be triggered by SARS$\mathrm{CoV}-2$ even in patients with mild or no respiratory symptoms with the acute viral infection, thereby necessitating correlation with COVID-19 testing to establish the association [40]. Imaging findings of SARS-CoV-2-related arthritis are nonspecific and include synovitis with power Doppler signal on ultrasound and synovial enhancement on MR imaging (Figs. 6 and 7) [30,31]. Features of inflammatory arthritis matching the underlying triggered condition, such as polyarticular synovitis and erosions, may be identified on imaging. Even in the absence of imaging findings characteristic of inflammatory arthritis, however, COVID-19 patients with acute arthritis may benefit from rheumatologic consultation.

\section{Soft tissues}

COVID-19 coagulopathy is associated with both thrombotic and bleeding manifestations, with development of disseminated intravascular coagulation in severe diseases [41]. There are several case reports of gangrene in COVID-19 patients, possibly due to thrombotic events and/or vasopressor medications administered for hemodynamic support [42-44]. The distal extremities are more susceptible to gangrene, particularly in patients with underlying diabetes or peripheral vascular disease. Imaging features of gangrene include skin ulcerations, T2 signal hyperintensity of soft tissues, and lack of enhancement of devitalized tissue. Superimposed infection, or "wet" gangrene, may result in soft tissue gas that can be identified on computed tomography (CT) or radiography. MR imaging is ideal for delineation of the extent of soft tissue necrosis and devitalization (Fig. 8) [45].
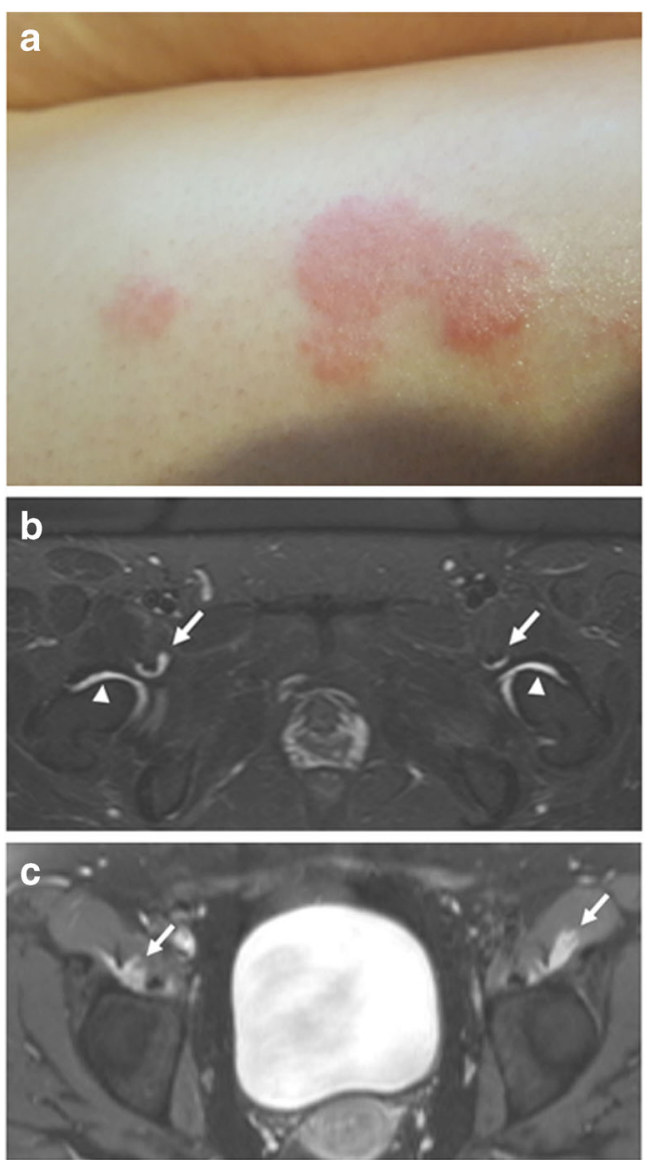

Fig. 7 A 30-year-old female with new-onset skin rashes and multiple arthralgias approximately 2 weeks after COVID-19. a Physical exam showed papules and plaques with areas of erythema, scaling, and lichenification over the extremities, axillae, and abdomen. Skin biopsy confirmed diagnosis of psoriasis. b Axial STIR and $\mathbf{c}$ post-contrast T1weighted fat-saturated images of the pelvis demonstrate mild bilateral hip synovitis (arrowheads) and iliopsoas bursitis (arrows). She was diagnosed with COVID-19-triggered psoriatic arthritis and treated with methotrexate, non-steroidal anti-inflammatory drugs, and corticosteroids

Bleeding complications can occur in COVID-19 patients secondary to virus-induced bleeding coagulopathy and/or anticoagulation therapy administered for treatment or prevention of the more commonly occurring thrombotic coagulopathy [41]. Hematomas may develop in subcutaneous soft tissues or within muscles. Complications from hematomas include compressive neuropathy, increased compartment pressure, and superimposed infection. CT, ultrasound, or MR imaging may be utilized for evaluation of hematomas, including diagnosis and follow-up imaging to ensure resolution (Figs. 9 and 10). Hematomas appear as heterogeneous hypoechoic fluid collections on ultrasound, with potential development of septa or calcifications in the chronic stage. MR imaging findings of hematomas include variable signal intensity due to different stages of blood degradation. In the subacute phase, a T1 hyperintense peripheral ring is distinctive [9].

"COVID toes" is a dermatologic manifestation of COVID19 that warrants mention given potential clinical request for 

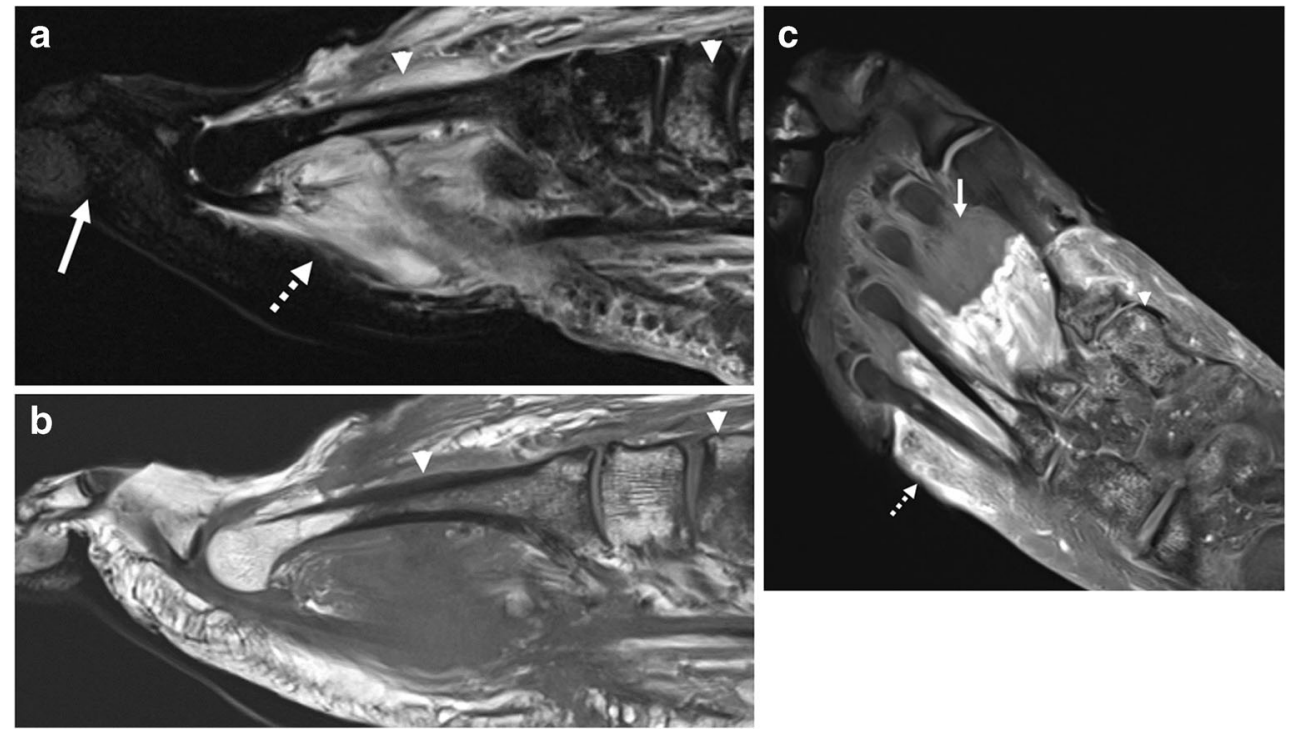

Fig. 8 A 57-year-old male with history of prostate cancer and prolonged hospitalization for COVID-19 with multi-organ failure requiring vasopressors. a Sagittal STIR image demonstrates soft tissue edema of the proximal foot (dashed arrow) but no edema distally (solid arrow). Heterogeneous bone marrow edema pattern (arrowhead) is seen on both
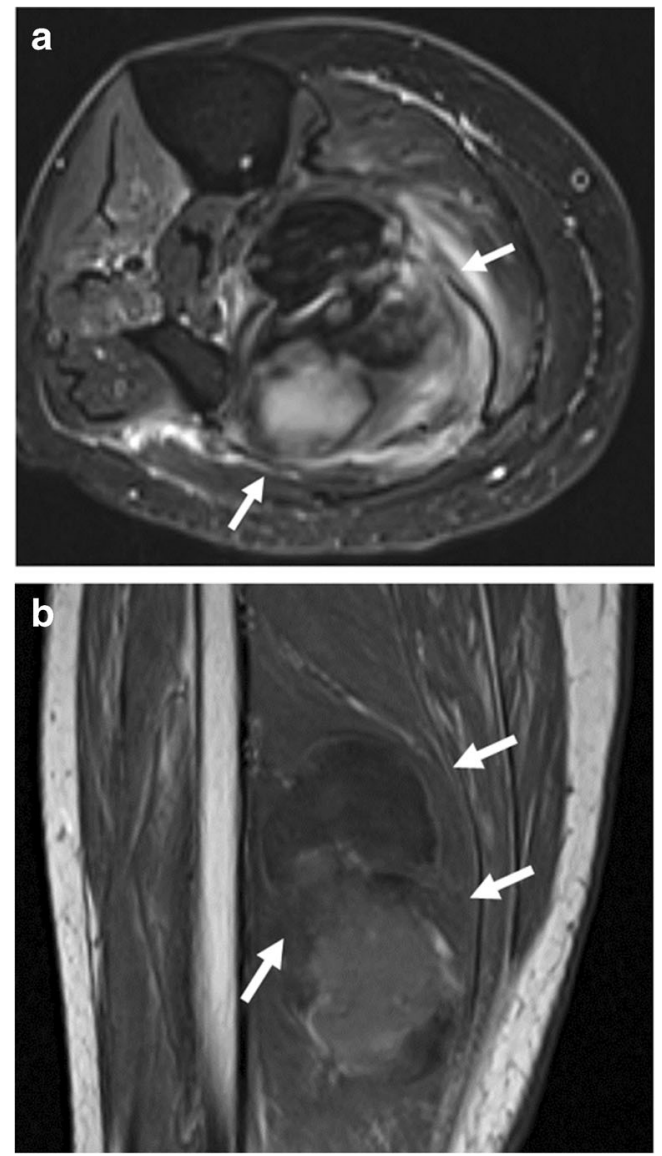

Fig. 9 A 71-year-old female with COVID-19 ARDS complicated by stroke and deep venous thrombosis, treated with systemic anticoagulation. She developed mass-like swelling of the leg. a Axial T2-weighted fat-saturated and b coronal T1-weighted images demonstrate an intramuscular hematoma (arrows)
STIR and b sagittal T1-weighted image. $\mathbf{c}$ Post-contrast T1-weighted fatsaturated image demonstrates soft tissue enhancement of the proximal foot (dashed arrow) with non-enhancing devitalized tissue distally (solid arrow), compatible with gangrene. Multifocal bone marrow edema pattern (arrowhead) is compatible with associated osteonecrosis
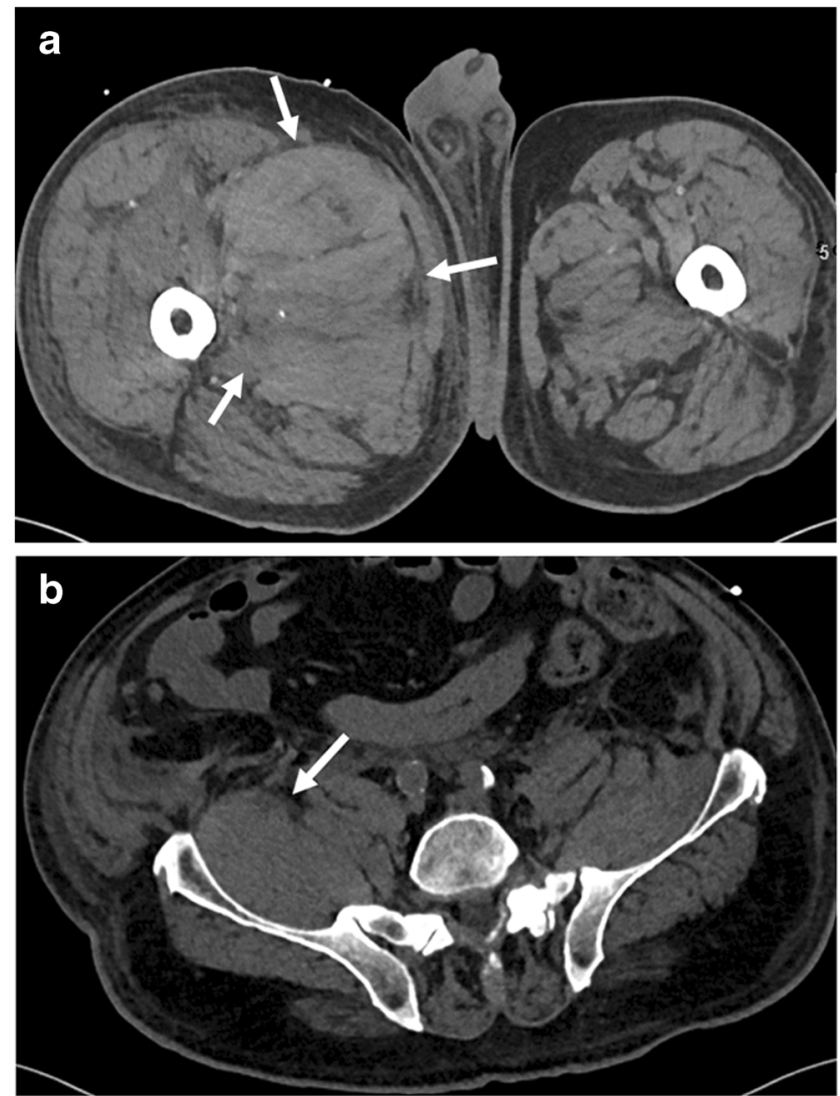

Fig. 10 A 76-year-old male with COVID-19 on therapeutic anticoagulation who developed right femoral and obturator neuropathies. a, b Axial CT demonstrates a large hematoma of the proximal right thigh musculature extending into the retroperitoneum along the right iliopsoas muscle (arrows) 
imaging. "COVID toes" is a chilblain-like phenomenon that manifests as erythema with vesicles or pustules, similar in appearance to frostbite and possibly due to a microvascular occlusive mechanism [3]. While there are currently no publications regarding imaging findings of "COVID toes," prior literature on the similar conditions of phalangeal microgeodic syndrome and Raynaud phenomenon have reported a distalto-proximal phalangeal bone marrow edema pattern on MRI $[46,47]$.

Of note, COVID-19 patients requiring prone positioning may develop soft tissue pressure injuries in uncommon ventral locations. Potential sites include the iliac crests, knees, anterior chest wall, and face. Obesity, male gender, and older age are risk factors for prone-related pressure injuries [19]. Imaging is generally not indicated unless there is clinical concern for superimposed infection potentially causing soft tissue abscess or osteomyelitis.

\section{Bones}

There is little current information regarding osseous complications of COVID-19. Critical illness, corticosteroid treatment, and virus-induced coagulopathy may contribute to the development of osteoporosis and osteonecrosis [48, 49]. Notably, the RECOVERY and REMAP-CAP clinical trials have recently demonstrated benefit of steroid treatment in COVID-19 patients [50, 51]. Imaging findings of osteonecrosis include osteosclerotic changes on radiography, serpiginous sclerosis with central lucency on CT, and lowsignal serpiginous line with central hyperintense line on MR imaging. Adjacent bone marrow edema pattern and articular surface collapse may be seen in later stages [45].

\section{Conclusion}

Multimodality imaging, including radiography, CT, ultrasound, and MR imaging, can play an important role in diagnosis and evaluation of COVID-19-related musculoskeletal pathology. Advances in imaging technology have improved diagnostic capabilities for even small structures such as peripheral nerves and in the setting of internal hardware with metal artifact reduction techniques [23, 52, 53]. Imaging can be utilized for initial diagnosis as well as for follow-up evaluation to assess recovery versus progression of disease. In cases where tissue sampling is necessary, imaging can provide procedural guidance. In some instances, musculoskeletal imaging may even suggest otherwise unrecognized SARS-CoV2 infection. Familiarity with incidence, etiology, and imaging findings of COVID-19-related musculoskeletal manifestations is important for radiologists in order to optimize imaging report interpretation and patient care. Although rare compared to other pulmonary and extra-pulmonary manifestations of SARS-CoV-2 infection, musculoskeletal disorders can have dire short- and long-term consequences.

Acknowledgements The authors would like to thank David C Botos, medical illustrator at Northwestern Memorial Hospital, Department of Radiology, for his original artwork (Figure 1).

\section{Declarations}

Conflict of interest The authors declare no competing interests.

\section{References}

1. Johns Hopkins University \& Medicine Coronavirus Resource Center. https://coronavirus.jhu.edu/map.html. Accessed 1/10/21

2. Revzin MV, Raza S, Warshawsky R, et al. Multisystem imaging manifestations of COVID-19. Part 1. Viral pathogenesis and pulmonary and vascular system complications. Radiographics. 2020;40(6):1574-99.

3. Revzin MV, Raza S, Srivastava NC, et al. Multisystem imaging manifestations of COVID-19. Part 2. From cardiac complications to pediatric manifestations. Radiographics. 2020;40(7):1866-92.

4. Wiersinga WJ, Rhodes A, Cheng AC, Peacock SJ, Prescott HC. Pathophysiology, transmission, diagnosis, and treatment of coronavirus disease 2019 (COVID-19): a review. JAMA. 2020;324(8): 782-93.

5. Paliwal VK, Garg RK, Gupta A, Tejan N. Neuromuscular presentations in patients with COVID-19. Neurol Sci. 2020;41(11):3039 56.

6. Keyhanian K, Umeton RP, Mohit B, Davoudi V, Hajighasemi F, Ghasemi M. SARS-CoV-2 and nervous system: from pathogenesis to clinical manifestation. J Neuroimmunol. 2020;350:577436.

7. Beydon M, Chevalier K, Al Tabaa O, et al. Myositis as a manifestation of SARS-CoV-2. Ann Rheum Dis. 2020:annrheumdis-2020217573. https://doi.org/10.1136/annrheumdis-2020-217573.

8. Wasserman PL, Way A, Baig S, Gopireddy DR. MRI of myositis and other urgent muscle-related disorders. Emerg Radiol. 2020;9: $1-13$.

9. Smitaman E, Flores DV, Mejía Gómez C, Pathria MN. MR imaging of atraumatic muscle disorders. Radiographics. 2018;38(2): 500-22.

10. Paganoni S, Amato A. Electrodiagnostic evaluation of myopathies. Phys Med Rehabil Clin N Am. 2013;24(1):193-207.

11. Yang T, Li Z, Jiang L, Xi X. Corticosteroid use and intensive care unit-acquired weakness: a systematic review and meta-analysis. Crit Care. 2018;22(1):187.

12. Cabañes-Martínez L, Villadóniga M, González-Rodríguez L, et al. Neuromuscular involvement in COVID-19 critically ill patients. Clin Neurophysiol. 2020;131(12):2809-16.

13. McCool FD, Manzoor K, Minami T. Disorders of the diaphragm. Clin Chest Med. 2018;39(2):345-60.

14. Guarracino F, Vetrugno L, Forfori F, et al. Lung, heart, vascular, and diaphragm ultrasound examination of COVID-19 patients: a comprehensive approach. J Cardiothorac Vasc Anesth. 2020: S1053-0770(20)30519-X. https://doi.org/10.1053/j.jvca.2020.06. 013.

15. Shi Z, de Vries HJ, Vlaar APJ, et al. Diaphragm pathology in critically Ill patients with COVID-19 and postmortem findings from 3 medical centers. JAMA Intern Med. 2021;181(1):122-4. https://doi.org/10.1001/jamainternmed.2020.6278. 
16. Caress JB, Castoro RJ, Simmons Z, et al. COVID-19-associated Guillain-Barré syndrome: the early pandemic experience. Muscle Nerve. 2020;62(4):485-91.

17. Koralnik IJ, Tyler KL. COVID-19: a global threat to the nervous system. Ann Neurol. 2020;88(1):1-11.

18. Mitry MA, Collins LK, Kazam JJ, Kaicker S, Kovanlikaya A. Parsonage-Turner syndrome associated with SARS-CoV2 (COVID-19) infection. Clin Imaging. 2020;72:8-10.

19. Le MQ, Rosales R, Shapiro LT, Huang LY. The down side of prone positioning: the case of a coronavirus 2019 survivor. Am J Phys Med Rehabil. 2020;99(10):870-2.

20. Malik GR, Wolfe AR, Soriano R, et al. Injury-prone: peripheral nerve injuries associated with prone positioning for COVID-19related acute respiratory distress syndrome. Br J Anaesth. 2020;125(6): $478-80$

21. Matschke J, Lütgehetmann M, Hagel C, et al. Neuropathology of patients with COVID-19 in Germany: a post-mortem case series. Lancet Neurol. 2020;19(11):919-29.

22. Montalvan V, Lee J, Bueso T, De Toledo J, Rivas K. Neurological manifestations of COVID-19 and other coronavirus infections: a systematic review. Clin Neurol Neurosurg. 2020;194:105921.

23. Ahlawat S, Chhabra A, Blakely J. Magnetic resonance neurography of peripheral nerve tumors and tumor like conditions. Neuroimaging Clin N Am. 2014;24(1):171-92.

24. Chhabra A, Madhuranthakam AJ, Andreisek G. Magnetic resonance neurography: current perspectives and literature review. Eur Radiol. 2018;28(2):698-707.

25. Brown JM, Yablon CM, Morag Y, Brandon CJ, Jacobson JA. US of the peripheral nerves of the upper extremity: a landmark approach. Radiographics. 2016;36(2):452-63.

26. Thawait SK, Chaudhry V, Thawait GK, et al. High-resolution MR neurography of diffuse peripheral nerve lesions. AJNR Am J Neuroradiol. 2011;32(8):1365-72.

27. Coppo A, Bellani G, Winterton D, et al. Feasibility and physiological effects of prone positioning in non-intubated patients with acute respiratory failure due to COVID-19 (PRON-COVID): a prospective cohort study. Lancet Respir Med. 2020;8(8):765-74. https:// doi.org/10.1016/S2213-2600(20)30268-X.

28. Schett G, Manger B, Simon D, Caporali R. COVID-19 revisiting inflammatory pathways of arthritis. Nat Rev Rheumatol. 2020;16(8):465-70.

29. Ciaffi J, Meliconi R, Ruscitti P, Berardicurti O, Giacomelli R, Ursini F. Rheumatic manifestations of COVID-19: a systematic review and meta-analysis. BMC Rheumatol. 2020;4:65.

30. Parisi S, Borrelli R, Bianchi S, Fusaro E. Viral arthritis and COVID19. Lancet Rheumatol. 2020;2(11):e655-7.

31. Danssaert Z, Raum G, Hemtasilpa S. Reactive arthritis in a 37-yearold female with SARS-CoV2 infection. Cureus. 2020;12(8):e9698.

32. Wendling D, Verhoeven F, Chouk M, Prati C. Can SARS-CoV-2 trigger reactive arthritis? Joint Bone Spine. 2020;27:105086.

33. Ono K, Kishimoto M, Shimasaki T, et al. Reactive arthritis after COVID-19 infection. RMD Open. 2020;6(2):e001350.

34. Schenker HM, Hagen M, Simon D, Schett G, Manger B. Reactive arthritis and cutaneous vasculitis after SARS-CoV-2 infection. Rheumatology (Oxford). 2020:keaa689. https://doi.org/10.1093/ rheumatology/keaa689.

35. Rodríguez Y, Novelli L, Rojas M, et al. Autoinflammatory and autoimmune conditions at the crossroad of COVID-19. J Autoimmun. 2020;114:102506.

36. Caso F, Costa L, Ruscitti P, et al. Could SARS-coronavirus-2 trigger autoimmune and/or autoinflammatory mechanisms in genetically predisposed subjects? Autoimmun Rev. 2020;19(5):102524.
37. Gokhale Y, Patankar A, Holla U, et al. Dermatomyositis during COVID-19 pandemic (a case series): is there a cause effect relationship? J Assoc Physicians India. 2020;68(11):20-4.

38. Bonometti R, Sacchi MC, Stobbione P, et al. The first case of systemic lupus erythematosus (SLE) triggered by COVID-19 infection. Eur Rev Med Pharmacol Sci. 2020;24(18):9695-7.

39. Mateu-Salat M, Urgell E, Chico A. SARS-COV-2 as a trigger for autoimmune disease: report of two cases of Graves' disease after COVID-19. J Endocrinol Investig. 2020;43(10):1527-8.

40. Novelli L, Motta F, Ceribelli A, Guidelli GM, et al. A case of psoriatic arthritis triggered by SARS-CoV-2 infection. Rheumatology (Oxford). 2021;60(1):e21-3. https://doi.org/10. 1093/rheumatology/keaa691.

41. Al-Samkari H, Karp Leaf RS, Dzik WH, et al. COVID-19 and coagulation: bleeding and thrombotic manifestations of SARSCoV-2 infection. Blood. 2020;136(4):489-500.

42. Zhang Y, Cao W, Xiao M, et al. Clinical and coagulation characteristics of 7 patients with critical COVID-2019 pneumonia and acro-ischemia. Zhonghua Xue Ye Xue Za Zhi. 2020;41(0):E006.

43. Novara E, Molinaro E, Benedetti I, Bonometti R, Lauritano EC, Boverio R. Severe acute dried gangrene in COVID-19 infection: a case report. Eur Rev Med Pharmacol Sci. 2020;24(10):5769-71.

44. Adekiigbe R, Ugbode F, Seoparson S, Katriyar N, Fetterman A. A 47-year-old Hispanic man who developed cutaneous vasculitic lesions and gangrene of the toes following admission to hospital with COVID-19 pneumonia. Am J Case Rep. 2020;21:e926886.

45. Leyva A, Cibulas A, Boron A, et al. Musculoskeletal faces of death: a diagnostic imaging review. Semin Roentgenol. 2019;54(2):190 202.

46. Smitaman E, Pereira BP, Huang BK, Zakhary MM, Fliszar E, Resnick DL. Abnormal bone marrow signal intensity in the phalanges of the foot as a manifestation of Raynaud phenomenon: a report of six patients. AJR Am J Roentgenol. 2016;207(6):1252-6. https:// doi.org/10.2214/AJR.16.16366.

47. Fujita A, Sugimoto H, Kikkawa I, Hyodoh K, Furuse M, Hoshino Y. Phalangeal microgeodic syndrome: findings on MR imaging. AJR Am J Roentgenol. 1999;173(3):711-2.

48. Disser NP, De Micheli AJ, Schonk MM, et al. Musculoskeletal consequences of COVID-19. J Bone Joint Surg Am. 2020;102(14):1197-204.

49. Zhang B, Zhang S. Corticosteroid-induced osteonecrosis in COVID-19: a call for caution. J Bone Miner Res. 2020;35(9): 1828-9.

50. The Writing Committee for the REMAP-CAP Investigators. Effect of hydrocortisone on mortality and organ support in patients with severe COVID-19: the REMAP-CAP COVID-19 corticosteroid domain randomized clinical trial. JAMA. 2020;324(13):1317-29.

51. RECOVERY Collaborative Group, Horby P, Lim WS, Emberson JR, et al. Dexamethasone in hospitalized patients with Covid-19 preliminary report. N Engl J Med. 2020:NEJMoa2021436. https:// doi.org/10.1056/NEJMoa2021436.

52. Sofka CM, Pavlov H. The history of clinical musculoskeletal radiology. Radiol Clin N Am. 2009;47(3):349-56.

53. Khodarahmi I, Fishman EK, Fritz J. Dedicated CT and MRI techniques for the evaluation of the postoperative knee. Semin Musculoskelet Radiol. 2018;22(4):444-56.

Publisher's note Springer Nature remains neutral with regard to jurisdictional claims in published maps and institutional affiliations. 